\title{
Estimation of Elasticity of Porous Rock Based on Mineral Composition and Microstructure
}

\author{
Zaobao Liu, ${ }^{1,2}$ Jianfu Shao, ${ }^{1,2}$ Weiya Xu, ${ }^{1}$ and Chong Shi ${ }^{1}$ \\ ${ }^{1}$ Geotechnical Research Institute, Hohai University, Nanjing 210098, China \\ ${ }^{2}$ Laboratory of Mechanics of Lille, University of Lille 1, 59655 Villeneuve d'Ascq, France \\ Correspondence should be addressed to Zaobao Liu; zaobaoliu@gmail.com
}

Received 14 May 2013; Revised 28 September 2013; Accepted 2 October 2013

Academic Editor: Jianqiao Ye

Copyright (c) 2013 Zaobao Liu et al. This is an open access article distributed under the Creative Commons Attribution License, which permits unrestricted use, distribution, and reproduction in any medium, provided the original work is properly cited.

\begin{abstract}
Estimation of elastic parameters of porous rock like the compressibility of sandstone is scientifically important and yet an open issue. This study illustrates the estimation of the elastic compressibility of sandstone (ECS) based on the assumption that the ECS is determined closely by the mineral composition and microstructures. In this study, 37 samples are collected to evaluate the estimations of the ECS obtained by different methods. The regression analysis is first implemented using the 37 samples. The results show that ECS exhibits linear relations with the rock minerals, pores, and applied compressive stress. Then the support vector machine (SVM) optimized by the particle swarm optimization algorithm (PSO) is examined to generate estimations of the ECS based on the mineral composition and microstructures. The SVM is trained with 30 samples to search for optimal parameters using the PSO, and thus the estimation model is established. Afterwards, this model is validated to give predictions of the left 7 samples. By comparison with the regression methods, the proposed strategy, that is, the PSO optimized SVM, performs much better on the training samples and shows a good capability in generating estimations of the ECS of the 7 testing samples based on the mineral composition and microstructures.
\end{abstract}

\section{Introduction}

The identification of elastic parameters of porous rock is one of the major problems in rock mechanics and so far an open issue. The elastic properties of porous rock show apparent variability from one project to another in practice. In the formulation of suitable constitutive models, the material parameters should be determined first from relevant experimental data in order to describe the mechanical behaviors of rock materials under different engineering contexts [1]. Classical deterministic approaches [2-4] have been firstly used to identify the physical properties of rock materials, generally based on laboratory experiments [5-9] and in situ tests $[10,11]$. However, these tests sometimes are difficult to be realized and may involve heavy costs. In this way, estimation of the coefficients related to the physical properties has also drawn much attention for feasibility and easiness in practice.

Approaching this issue, many techniques have been proposed for the estimation of elastic parameters of porous rock material [4, 12-18]. In these techniques, the elastic parameters are thought to be closely related to some other indexes that are easily to be determined. However, these conventional methods, for example, the empirical equations, have very poor generalization ability in estimation. This is doomed due to the insufficiency of these methods to account for the uncertain relationships between the elastic parameters and the related indexes such as the rock minerals and microstructures.

In order to better take into account the uncertainties in the determination of elastic parameters of rock materials, various soft computing methods have been introduced to approach this problem in the past decades [19]. These methods provide a new way for the description of elastic parameters of rock materials since with such approaches it becomes possible to learn some disciplines among the related rock parameters from the relevant data obtained. In this manner, if similar positive results can be found, expensive experimental identification procedures can be avoided. Towards this issue, some valuable results have been obtained in some previous works by using the neural networks and regression 
techniques [20-25]. In these works, both the laboratory tests and field measurements have been used for estimating the elastic parameters. However, there is a common shortcoming for both the field tests and the laboratory tests. They cannot consider all the physical and mineral parameters such as the mineral composition, the particle size, and the distribution of voids. And what is more, as mentioned above, they are expensive to be realized in some cases. For the simple neural networks, they have some shortcomings all the same, such as the local solution, weak generalization ability, and high computational expense. In this way, more effective methods are still in need to approach the estimation of the elastic parameters of the porous rock materials.

In this paper, we illustrate estimation of the elastic parameters of sandstone (the one common material in geotechnical engineering and earth science) according to their mineral compositions and microstructural properties using the SVM model $[26,27]$. We apply the particle swarm optimization algorithm (PSO) to optimize the SVM model parameters which have been proved to have a significant effect on the model performance [28]. We demonstrate the applicability and reliability of this method noted as the PSOSVM for estimation of rock elastic compressibility based on the experimental data of the rock mineral compositions and microstructure features as well as the loading pressure. The other elastic parameters of porous rock can be estimated using the strategy in a similar way and are thus not discussed in this paper.

\section{Material and Method}

2.1. Rock Elastic Compressibility and Experimental Data. Compressibility is a measure of the relative volume change of material as a response to a stress (or hydrostatic pressure) change under certain conditions. The measurement of rock compressibility is accomplished through measuring the change of pore volume versus pore pressure. The rock compressibility usually has an unreasonable deviation from its true value for the reason that the measurement is invisibly affected by many uncertain parameters.

Rock compressibility is the volume shrunken feature of rocks under pressure, and is reflected by the compressibility coefficients. The compressibility coefficients of the rock are closely related to the ambient pressure, as well as the fracture distribution, the mineral and its proportion, density, and void ratio. The beginning work on rock compressibility that is widely used today is done by Hall [29] in 1953. He developed a graph of the rock compressibility versus porosity by statistical analysis of laboratory experiments, which is called Hall's plot today and simulated by some empirical formula. Then a similar fundamental work has been done by Newman [30]. He obtained a similar trend of rock compressibility with porosity to Hall's plot for both the consolidated sandstone and limestone.

However, Hall's plot, in some cases, shows a logically confusion relation between the compressibility and porosity in rocks. According to Hall's plot, the compressibility decreases as rock porosity increases. Extremely tight rocks have an abnormally high compressibility [31]. In fact, tight rocks are less compressible than loose rocks and should have a smaller compressibility. Moreover, the rock compressibility by Hall's plot is usually larger than that of the reservoir liquids in the normal range of reservoir porosity. Thus, Hall's plot is not sufficient enough in every case. Also, Hall's plot gives the same compressibility value for rocks of different lithology if only they have the same porosity regardless of their different rigidity. That is to say, Hall's plot does not consider the effect of rock lithology and minerals composing the rocks. Some other discussions on the rock compressibility can also be found in the pieces of literature [32-36].

Experiments have been done on sandstones for discovering the relationships between the compressibility coefficients and their mineral compositions, voids, and other parameters [3]. As stated in the work, the samples used in the experiments are mostly clean quartz arenites, subarkoses, and argillaceous quartz arenites, in which kaolinite is the dominant porefilling mineral. Total porosity is divided into three types which are the intergranular (equidimensional, size comparable to grains), the connective (tabular or tubular shaped), and the micro (less than a few microns in size) porosity on the basis of point counting SEM images. The micro pores occur within aggregates of clay. The ranges of porosity of samples are total porosity, $5-31 \%$ of whole sample; intergranular porosity, $24-76 \%$ of total porosity; connective porosity, 4$25 \%$ of total porosity; microporosity, $10-63 \%$ of total porosity. Empirical equations of calculating rock compressibility are mainly based on the rock porosity and cannot take into account the effects of all the associated parameters.

The experimental results are rearranged as shown in Table 1 where the three coefficients $a_{A}, a_{B}$, and $a_{C}$ are the elastic linear compressibility measured by gauges settled in three orthogonal directions. In all, 37 samples of sandstone are used in this study and each sample has 11 features. The box graph of each parameter of all the sandstone samples is shown in Figure 1.

The parameter values of the samples are shown in two subfigures (Figures 1(a) and 1(b)) in order to well illustrate their statistical aspects due to the differences of their value ranges. The horizontal axis of Figure 1 lists the parameter names and the vertical axis denotes the parameter values without units. The small circles (o) and the stars $(*)$ in Figure 1 exhibit the "outliers" produced by the box graph based on the statistical features of the dataset. The numbers beside the circle and star markers are the sample numbers listed in the first column of Table 1 . They are the test parameter values which are not in the statistical range of the box graph. In this manner, these "outliers" do not necessarily mean that they are true outliers and should be removed from analysis of the dataset. Nevertheless, the box graph manifests that some parameters in the dataset have several "outliers" which indicate the moderate quality of statistical consistency of the dataset.

2.2. Support Vector Machine. The support vector machines, also known as the support vector networks [26], are supervised learning models with associated learning algorithms which deal with data and recognize patterns and are mainly used for classification and regression analysis. 


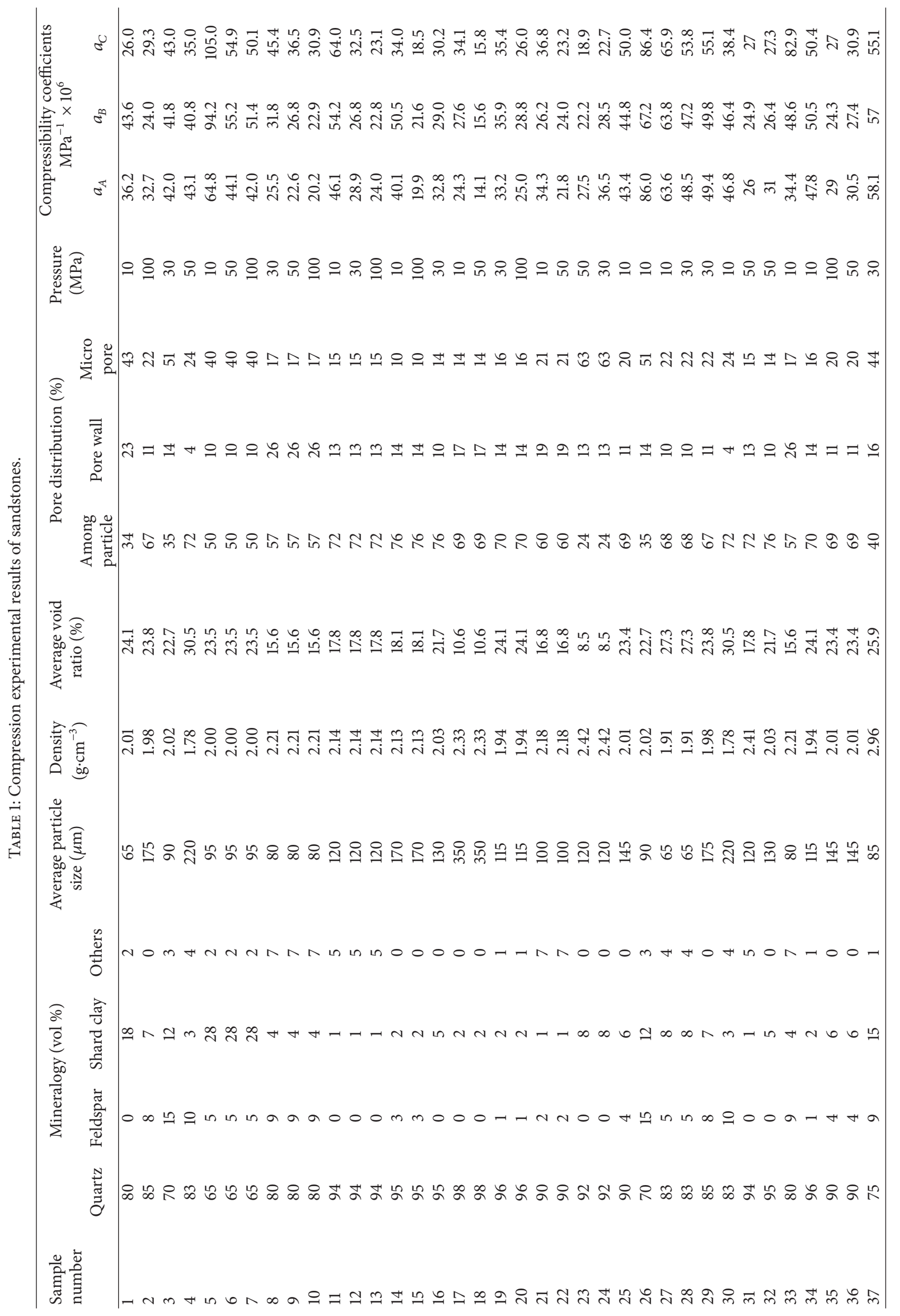




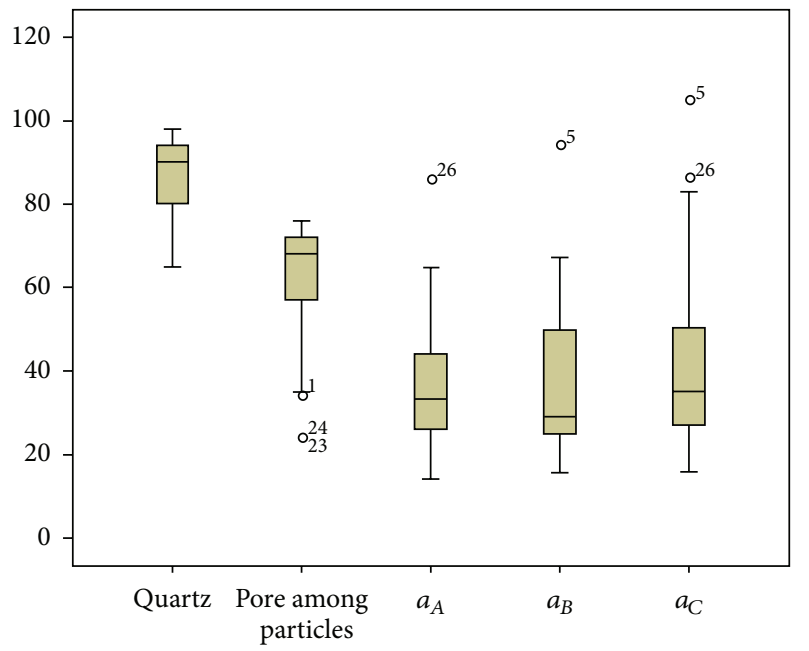

(a)

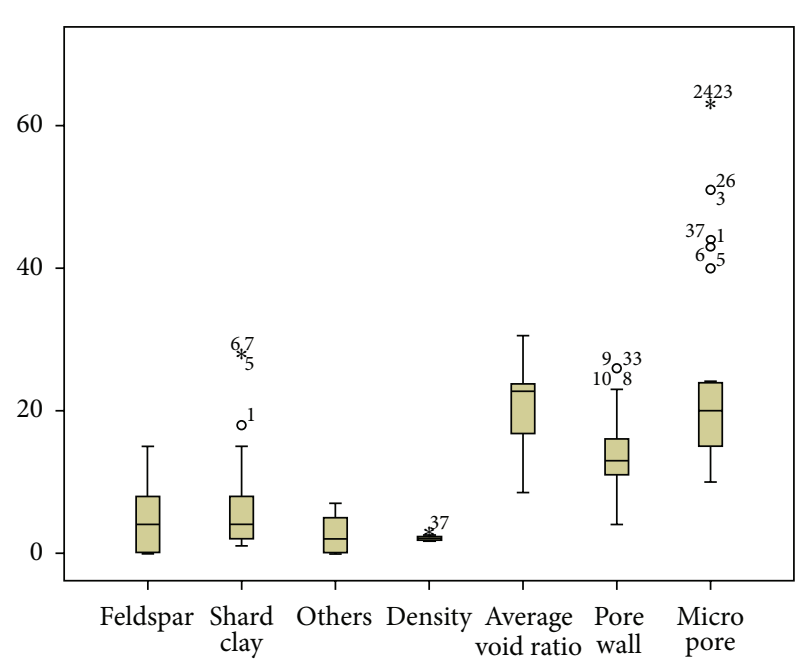

(b)

FIGURE 1: Box graph of the properties of sandstone experiment data (without unit).

Given the 30 training samples in Table 1 denoted by $(X, Y)=\left(x_{i}, y_{i}\right)_{i=1}^{n}$, here $x_{i}$ is the $i$ th sample with 11 parameters values (e.g., the mineral type and the density); $y_{i}$ is the three compressibility coefficients $\left(a_{A}, a_{B}\right.$, and $\left.a_{C}\right)$ of the $i$ th sample; $n$ is the sample number $(n=30)$. The SVMs make a mapping of the samples with a linear regression function

$$
y=f(x)=w \cdot \phi(x)+b
$$

where $w$ is the weight vector, $b$ is bias, and $\phi(x)$ is the nonlinear mapping from the input space to output space. The SVMs can efficiently perform nonlinear mappings using what is called the kernel trick, implicitly mapping their inputs into high-dimensional feature spaces.

Suppose that all the samples can be mapped well with a linear function with precision $\varepsilon$. Considering the true mapping errors, the nonnegative slack variables $\xi_{i}$ and $\xi_{i}^{*}$ can be introduced. Thus, the problem can be transformed with the inequalities

$$
\begin{gathered}
y_{i}-w \phi\left(x_{i}\right)-b \leq \varepsilon+\xi_{i} \\
\phi\left(x_{i}\right) w+b-y_{i} \leq \varepsilon+\xi_{i}^{*}, \quad i=1,2, L, n .
\end{gathered}
$$

The purpose of SVM training is to minimize the following function:

$$
\phi\left(w, \xi_{i}, \xi_{i}^{*}\right)=\frac{1}{2}\left(w w^{T}\right)+C \sum_{i=1}^{n}\left(\xi_{i}+\xi_{i}^{*}\right),
$$

where the constant $C>0$ is the penalty parameter denoting the punishing level of the samples with errors over $\varepsilon$. Therefore, the problem can be rewritten as

$$
\begin{array}{cl}
\max \quad & \left\{\frac{1}{2} \sum_{i=1}^{n} \sum_{j=1}^{n}\left(\alpha_{i}-\alpha_{i}^{*}\right)\left(\alpha_{j}-\alpha_{j}^{*}\right) K\left(x_{i}, x_{j}\right)\right. \\
& \left.-\varepsilon \sum_{i=1}^{n}\left(\alpha_{i}-\alpha_{i}^{*}\right)+\sum_{i=1}^{n} y_{i}\left(\alpha_{i}-\alpha_{i}^{*}\right)\right\}, \\
\text { S.t. } \quad & \sum_{i=1}^{n}\left(\alpha_{i}-\alpha_{i}^{*}\right)=0 \quad\left(\alpha_{i}, \alpha_{i}^{*} \in[0, c]\right),
\end{array}
$$

where $K\left(x_{i}, x_{j}\right)=\phi\left(x_{i}\right) \phi\left(x_{j}\right)$ is the kernel function. There are many commonly used kernel functions, like the multinomial kernel, the sigmoid kernel, and the RBF kernel.

In this way, the prediction model of the SVMs can be obtained as follows:

$$
f(x)=\sum_{i=1}^{n}\left(\alpha_{i}-\alpha_{i}^{*}\right) K\left(x_{i}, x\right)+b .
$$

2.3. Particle Swarm Optimization. The particle swarm optimization (PSO) algorithm is proposed for searching the optimal solution in complex space by the collaboration and competition among particle individuals. It is a population based stochastic optimization technique inspired by social behavior of bird flocking or fish schooling, developed by Eberhart and Kennedy in 1995 [37]. The PSO simulates the foraging behavior of birds. Each solution in the optimizing problem is looked as a bird or a particle in the algorithm in the searching space. The goodness of a particle is evaluated by the value of the fitness function. Each particle keeps track of its coordinates in the problem space which are associated with the best solution (fitness) it has achieved so far. The fitness value is also stored. This value is called $P_{\text {best }}$. Another "best" value that is tracked by the particle swarm optimizer is the best value, obtained so far by any particle in the neighbors of the particle. This location is called $L_{\text {best }}$. The best value is a global best and is called $G_{\text {best }}$ when a particle takes all the population as its topological neighbors.

The velocity of the particle $i$ in the $n$ dimensional space is denoted as $v_{i}=\left\{v_{i 1}, v_{i 2}, \ldots, v_{i n}\right\}$. The corresponding location is $x_{i}=\left\{x_{i 1}, x_{i 2}, \ldots, x_{i n}\right\}$; the best solution of the particle is $P_{\text {best }}=\left\{p_{i 1}, p_{i 2}, \ldots, p_{i n}\right\}$; the global best solution is $G_{\text {best }}=$ $\left\{g_{1}, g_{2}, \ldots, g_{n}\right\}$.

The particle swarm optimization concept consists in, at each time step, changing the velocity of (accelerating) each particle toward its $P_{\text {best }}$ and $L_{\text {best }}$ locations (local version of PSO). Acceleration is weighted by a random term, with separate random numbers being generated for acceleration 
TABLE 2: Model summary of stepwise regression for $a_{A}$.

\begin{tabular}{|c|c|c|c|c|c|c|c|c|c|}
\hline \multirow{2}{*}{ Model } & \multirow{2}{*}{$R$} & \multirow{2}{*}{$R$ square } & \multirow{2}{*}{ Adjusted $R$ square } & \multirow{2}{*}{ Std. error of the estimate } & \multicolumn{5}{|c|}{ Change statistics } \\
\hline & & & & & $R$ square change & $F$ change & $d f 1$ & $d f 2$ & Sig. $F$ change \\
\hline 1 & $.542^{\mathrm{a}}$ & .294 & .274 & 12.53587 & .294 & 14.560 & 1 & 35 & .001 \\
\hline 2 & $.713^{\mathrm{b}}$ & .509 & .480 & 10.60497 & .215 & 14.906 & 1 & 34 & .000 \\
\hline 3 & $.834^{\mathrm{c}}$ & .695 & .668 & 8.47732 & .186 & 20.208 & 1 & 33 & .000 \\
\hline
\end{tabular}

toward $P_{\text {best }}$ and $L_{\text {best }}$ locations. PSO updates the velocity and location of the particles with the following equation:

$$
\begin{gathered}
v_{i}^{k+1}=w v_{i}^{k}+c_{1} r(\cdot)\left(P_{\text {best }}-x_{i}^{k}\right)+c_{2} r(\cdot)\left(G_{\text {best }}-x_{i}^{k}\right), \\
x_{i}^{k+1}=x_{i}^{k}+v_{i}^{k+1},
\end{gathered}
$$

where $k$ is the iteration number; $w$ is the inertia weight; $r(\cdot)$ is a random constant uniformly distributed in the interval $(0,1)$; $c_{1}$ and $c_{2}$ are the learning coefficients.

2.4. PSO Optimized SVM. The values of the penalty parameter $C$ and the kernel parameters affect directly the model performance in the SVM modeling. Due to the fast and global optimizing features of PSO, it is applied to optimize the parameters in the SVM modeling. The implementation is done in the following steps.

(a) Initialize the parameters in PSO, including the particle size, the iteration number, the inertia weight $w$, and the learning coefficients $c_{1}$ and $c_{2}$.

(b) Determine the range of the optimized parameters and specify $P_{\text {best }}$ and $G_{\text {best }}$.

(c) Define the fitness function

$$
f(x)=\sum_{i=1}^{n} \frac{\left|u_{i}-u_{i}^{*}\right|}{u_{i}}
$$

where $u_{i}$ is the observed value of the $i$ th sample; $u_{i}^{*}$ is the predicted value of SVM; $i=1,2, \ldots, n$ is the sample numbers.

(d) Calculate the fitness value of every particle and compare this value with the $P_{\text {best }}$ (the best fitness value of its ever best location $L_{\text {best }}$ ). If this value is better than $P_{\text {best }}$, then update $L_{\text {best }}$ with the new location.

(e) Compare $P_{\text {best }}$ with $G_{\text {best }}$. If $P_{\text {best }}$ is better than $G_{\text {best }}$, then renew $G_{\text {best }}$ with $P_{\text {best }}$.

(f) Check whether the fitness value or the iteration number is satisfied with the end condition of the algorithm. If not, update the location and velocity of the particle with (6) or exit and output the results of the coefficients.

(g) Set up the optimized SVM model for modeling in (5) with the optimized parameters.

The implementation of the estimation is given in Figure 2.

\section{Results and Discussions}

3.1. Regression Analysis. We first apply the linear stepwise regression method [38] to analyze the problem. In the stepwise linear regression, the forward method is used to remove the variables in the regression models. The stepping method criteria are the probability $P\left(F\right.$ to remove $\left.X_{k}\right)>0.10$ to determine whether a parameter $X_{k}$ is removed. If the inequality does not hold, no variable is removed from the model. If there are no independent variables currently entered in the model or if no entered variable is to be removed, choose $X_{k}$ such that $P\left(F\right.$ to remove $\left.X_{k}\right)$ is maximum. A parameter $X_{k}$ is entered if $P\left(F\right.$ to remove $\left.X_{k}\right)<0.05$. If the inequality does not hold, no variable is entered. At each step, all eligible variables are considered for removal and entry [39].

The results of the stepwise regression models are summarized in Table 2 to Table 4, respectively, for the three coefficients $a_{A}, a_{B}$, and $a_{C}$. Three predictors are generalized for each coefficient in the regression. It is obvious that the predictor $a$ and predictor $b$ in each model are not physically meaningful at all because the two predictors only retain no more than two potential parameters to explain the compressive coefficients, which is obviously unmeaning. The $R$ square and adjusted $R$ square values are all less than 0.70 for model 3(predictor $c$ ) in Table 2 to Table 4. This implies that the compressive coefficients do not have a strict linear relation to the associated parameters. The value "sig. $F$ change" in Tables 2, 3, and 4 shows that the derived regression models are statistically significant (less than 0.005). In short, the stepwise regression can only perform moderately in modeling these sand rock samples.

The remaining parameters are the mineral quartz, the pressure, and the pore wall for the coefficients $a_{A}$ and $a_{B}$ in predictor $c$. For the coefficient $a_{C}$, the remaining parameters are quartz, the pressure and the pore among particles in Predictor $c$. That is to say those parameters related to the rock minerals, applied pressure, and pores are remained exclusively in the stepwise regression results. At this point, the rock compressibility coefficients can be thought to have linear relations with the rock minerals, loading pressure, and pores which can be used to interpret the characteristics of the rock compressibility coefficients.

3.2. PSO-SVM Analysis. According to the experimental results, we assume that the minerals of the rock, average size of crystalline particle, interspace distribution, average void ratio, the density of rock, and the pressure of the test specimen are the potential influencing parameters for compressibility coefficients of sand rock materials. We utilize the PSO-SVM 


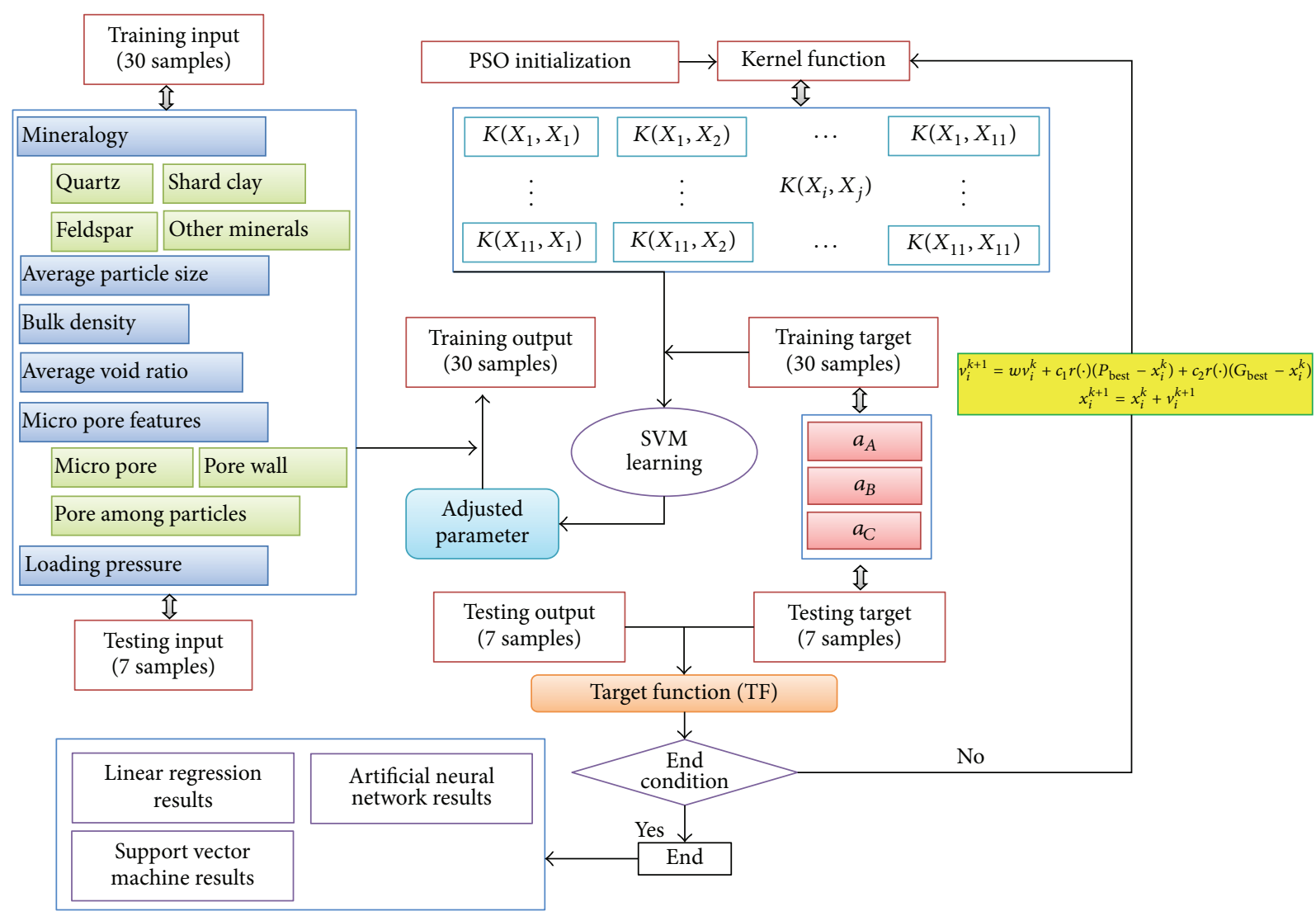

FIGURE 2: Implementation of estimation of rock elastic compressibility using PSO-SVM.

TABLE 3: Model summary of stepwise regression for $a_{B}$.

\begin{tabular}{lccccccccc}
\hline \multirow{2}{*}{$\begin{array}{c}\text { Model } \\
\end{array}$} & \multirow{2}{*}{$R$ square } & \multirow{2}{*}{ Adjusted $R$ square } & \multicolumn{2}{c}{ Std. error of the estimate } & \multicolumn{4}{c}{ Change statistics } \\
\hline 1 & $.608^{\mathrm{a}}$ & .369 & .351 & 13.44219 & .369 & 20.485 & 1 & 35 & .000 \\
2 & $.756^{\mathrm{b}}$ & .571 & .546 & 11.24635 & .202 & 16.002 & 1 & 34 & .000 \\
3 & $.825^{\mathrm{c}}$ & .680 & .651 & 9.85276 & .109 & 11.298 & 1 & 33 & .002 \\
\hline
\end{tabular}

${ }^{\mathrm{a}}$ Predictor: (constant), quartz; ${ }^{\mathrm{b}}$ predictor: (constant), quartz, pressure; ${ }^{\mathrm{c}}$ predictor: (constant), quartz, pressure, and pore wall; ${ }^{\mathrm{d}}$ dependent variable: $a_{B}$.

TABLE 4: Model summary of stepwise regression for $a_{C}$.

\begin{tabular}{cccccccccc}
\hline \multirow{2}{*}{ Model } & \multirow{2}{*}{$R$} & \multirow{2}{*}{$R$ square } & Adjusted $R$ square & Std. error of the estimate & \multicolumn{4}{c}{ Change statistics } \\
& & & & & $R$ square change & $F$ change & $d f 1$ & $d f 2$ & Sig. $F$ change \\
\hline 1 & $.627^{\mathrm{a}}$ & .393 & .375 & 15.82344 & .393 & 22.641 & 1 & 35 & .000 \\
2 & $.777^{\mathrm{b}}$ & .604 & .581 & 12.95912 & .212 & 18.182 & 1 & 34 & .000 \\
3 & $.816^{\mathrm{c}}$ & .667 & .636 & 12.07530 & .062 & 6.159 & 1 & 33 & .018 \\
\hline
\end{tabular}

${ }^{a}$ Predictor: (constant), quartz; ${ }^{b}$ predictor: (constant), quartz, pressure; ${ }^{c}$ predictor: (constant), quartz, pressure, and pore among particles; ${ }^{\mathrm{d}}$ dependent variable: $a_{\mathrm{C}}$.

to map the relations between the compressibility coefficients and their potential influencing parameters and compare the results with those of the (ANN) [20] and simple SVM models. The first 30 samples listed in Table 1 are used as training samples to establish the models and the last 7 samples are used for testing generalization ability of produced models. The structures of SVM are shown in Figure 2 for the estimation of elastic compressibility of sandstone.
The predicted rock compressibility coefficients of the tested samples are shown in Table 5 for all the introduced techniques. Based on these results, the predictive performance of these approaches is illustrated in Figure 3 to Figure 5 for $a_{A}, a_{B}$, and $a_{C}$, respectively. The linear trend lines in the figures show the correlation between the observed value and predicted value. The results of different approaches are shown with different markers and different colors. The $R^{2}$ values 
TABLE 5: Measured and predicted rock compressibility coefficients.

\begin{tabular}{lccccccccccccc}
\hline \multirow{2}{*}{ Sample number } & \multicolumn{3}{c}{ Measured } & \multicolumn{3}{c}{ ANN prediction } & \multicolumn{3}{c}{ SVM prediction } & \multicolumn{3}{c}{ PSO-SVM prediction } \\
& $a_{A}$ & $a_{B}$ & $a_{C}$ & $a_{A}$ & $a_{B}$ & $a_{C}$ & $a_{A}$ & $a_{B}$ & $a_{C}$ & $a_{A}$ & $a_{B}$ & $a_{C}$ \\
\hline 1 & 26.0 & 24.9 & 27.0 & 25.0 & 25.2 & 24.3 & 23.2 & 23.9 & 24.8 & 27.1 & 24.6 & 28.5 \\
2 & 31.0 & 26.4 & 27.3 & 30.0 & 29.4 & 25.6 & 26.8 & 23.4 & 25.0 & 32.1 & 27.9 & 28.0 \\
3 & 34.4 & 48.6 & 82.9 & 59.9 & 65.1 & 87.0 & 30.5 & 34.1 & 49.8 & 42.2 & 54.4 & 79.6 \\
4 & 47.8 & 50.5 & 50.4 & 41.9 & 39.7 & 43.9 & 41.4 & 43.8 & 43.8 & 41.9 & 55.2 & 57.7 \\
5 & 29.0 & 24.3 & 27.0 & 28.1 & 26.8 & 24.1 & 27.5 & 22.8 & 25.6 & 30.1 & 26.1 & 26.5 \\
6 & 30.5 & 27.4 & 30.9 & 34.8 & 33.0 & 32.3 & 33.3 & 29.6 & 35.6 & 31.2 & 31.5 & 32.1 \\
7 & 58.1 & 57 & 55.1 & 54.9 & 61.6 & 69.9 & 40.2 & 43.8 & 43 & 55.1 & 60.6 & 62.9 \\
\hline
\end{tabular}

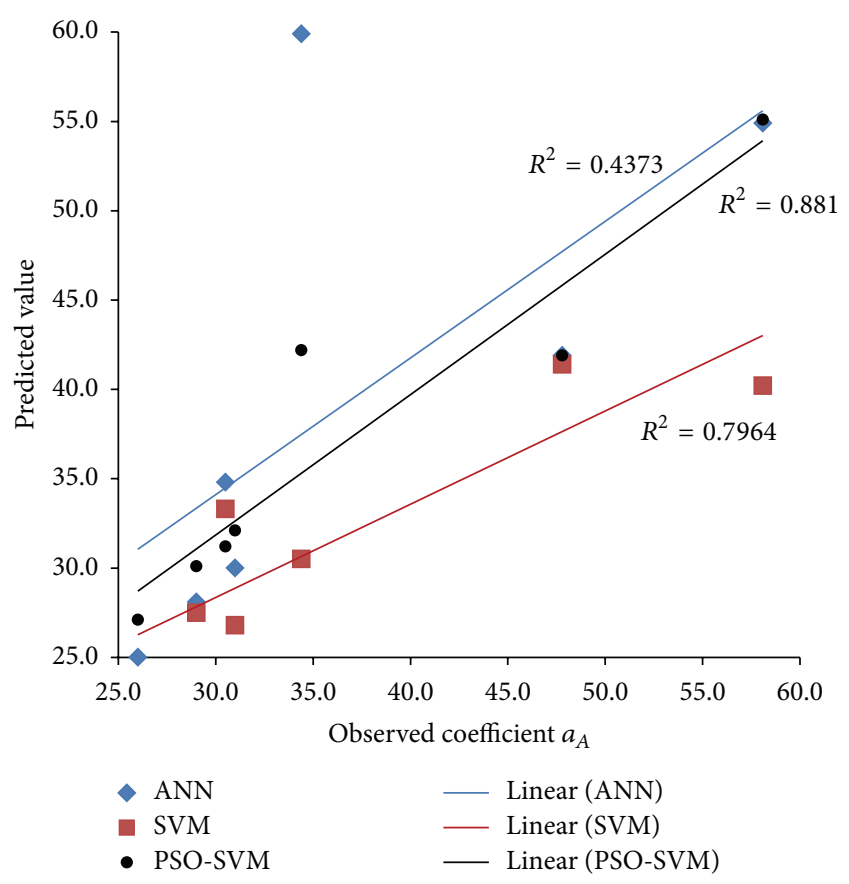

Figure 3: Predictive performance of $a_{A}$.

imply directly the predictive performances. The larger the $R^{2}$ value is, the better the corresponding approach performs. It is obvious that the PSO-SVM approach performs best among these techniques regarding the prediction of these three coefficients. Also, the $R^{2}$ value in Figure 3 is much smaller than that in Figures 4 and 5, which indicates that these approaches cannot generate as good results of $a_{A}$ as those of $a_{B}$ or $a_{C}$.

3.3. Predicted Error Comparison. In order to evaluate the model performance, the absolute error function and relative error function are used to compare the predictive results of the various methods used for modeling the sand rock samples. Consider

Absolute error $=\mid$ Predicted value - observed value $\mid$,

\section{Relative error}

$$
=\frac{\mid \text { Predicted value }- \text { observed value } \mid}{\text { Observed value }} \times 100 \% \text {. }
$$

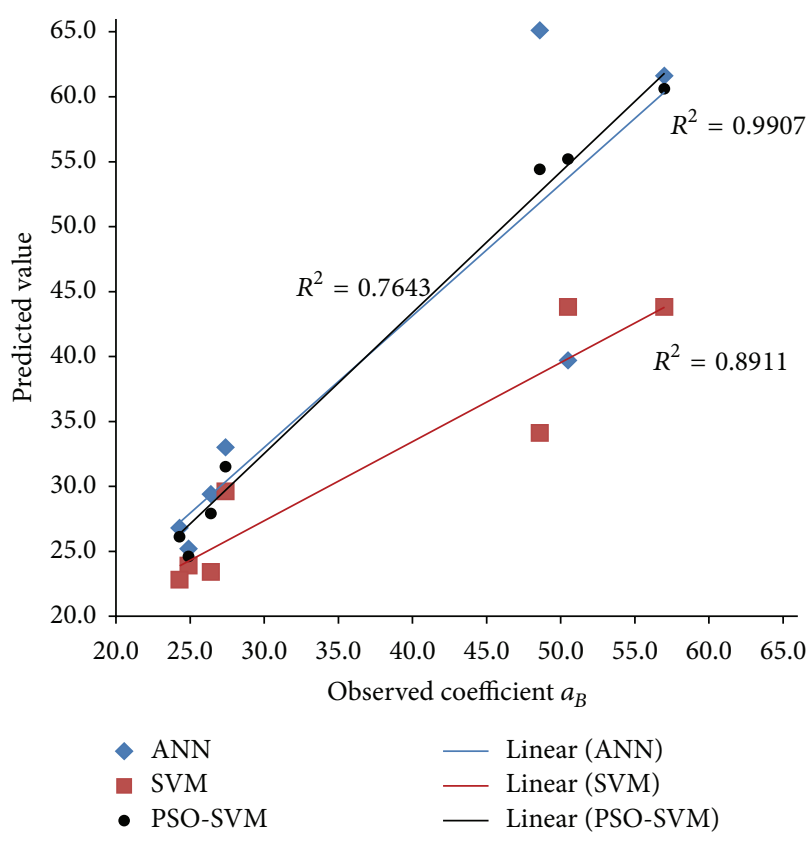

FIgURE 4: Predictive performance of $a_{B}$.

The absolute prediction errors $(\mathrm{AE})$ and relative prediction errors (RE) are given in Table 6, respectively. The average prediction errors of all the approaches are shown in Figure 6 for the three rock compressibility coefficients. From these results, it is interesting to see that the prediction errors of the test samples 3, 4, and 7 are much larger than those of the other test samples in all the used techniques. Generally, the prediction errors of ANN approach are the biggest and that of the PSO-SVM approach is the smallest. The average prediction error bar in Figure 6 has shown this more apparently.

The prediction errors of the PSO-SVMs are nearly half of those of the ANNs. Despite this, it does not necessarily mean that the ANNs are not suitable for estimating the rock compressibility coefficients. In the modeling of ANNs approach, only a pair of initialized parameter values of the networks is given and no optimization techniques are applied to find optical parameters. In the simple SVM modeling, again, no optimizing algorithms are applied to obtain the optical penalty parameter and kernel parameters. While in the PSO-SVM modeling, the parameters in the SVM model 
TABLE 6: Relative prediction error of different models for the test samples.

\begin{tabular}{|c|c|c|c|c|c|c|c|c|c|c|c|c|c|c|c|c|c|c|}
\hline \multirow{3}{*}{ Test sample no. } & \multicolumn{6}{|c|}{ ANN prediction } & \multicolumn{6}{|c|}{ SVM prediction } & \multicolumn{6}{|c|}{ PSO-SVM prediction } \\
\hline & \multicolumn{2}{|c|}{$a_{A}$} & \multicolumn{2}{|c|}{$a_{B}$} & \multicolumn{2}{|c|}{$a_{\mathrm{C}}$} & \multicolumn{2}{|c|}{$a_{A}$} & \multicolumn{2}{|c|}{$a_{B}$} & \multicolumn{2}{|c|}{$a_{C}$} & \multicolumn{2}{|c|}{$a_{A}$} & \multicolumn{2}{|c|}{$a_{B}$} & \multicolumn{2}{|c|}{$a_{C}$} \\
\hline & $\mathrm{AE}$ & $\mathrm{RE}$ & $\mathrm{AE}$ & $\mathrm{RE}$ & $\mathrm{AE}$ & $\mathrm{RE}$ & $\mathrm{AE}$ & $\mathrm{RE}$ & $\mathrm{AE}$ & $\mathrm{RE}$ & $\mathrm{AE}$ & $\mathrm{RE}$ & $\mathrm{AE}$ & $\mathrm{RE}$ & $\mathrm{AE}$ & $\mathrm{RE}$ & $\mathrm{AE}$ & $\mathrm{RE}$ \\
\hline 1 & 1.0 & 3.8 & 0.3 & 1.2 & 2.7 & 10.0 & 2.8 & 10.8 & 1.0 & 4.0 & 2.2 & 8.1 & 1.1 & 4.2 & 0.3 & 1.2 & 1.5 & 5.6 \\
\hline 2 & 1.0 & 3.2 & 3.0 & 11.4 & 1.7 & 6.2 & 4.2 & 13.5 & 3.0 & 11.4 & 2.3 & 8.4 & 1.1 & 3.5 & 1.5 & 5.7 & 0.7 & 2.6 \\
\hline 3 & 25.5 & 74.1 & 16.5 & 34.0 & 4.1 & 4.9 & 3.9 & 11.3 & 14.5 & 29.8 & 33.1 & 39.9 & 7.8 & 22.7 & 5.8 & 11.9 & 3.3 & 4.0 \\
\hline 4 & 5.9 & 12.3 & 10.8 & 21.4 & 6.5 & 12.9 & 6.4 & 13.4 & 6.7 & 13.3 & 6.6 & 13.1 & 5.9 & 12.3 & 4.7 & 9.3 & 7.3 & 14.5 \\
\hline 5 & 0.9 & 3.1 & 2.5 & 10.3 & 2.9 & 10.7 & 1.5 & 5.2 & 1.5 & 6.2 & 1.4 & 5.2 & 1.1 & 3.8 & 1.8 & 7.4 & 0.5 & 1.9 \\
\hline 6 & 4.3 & 14.1 & 5.6 & 20.4 & 1.4 & 4.5 & 2.8 & 9.2 & 2.2 & 8.0 & 4.7 & 15.2 & 0.7 & 2.3 & 4.1 & 15.0 & 1.2 & 3.9 \\
\hline 7 & 3.2 & 5.5 & 4.6 & 8.1 & 14.8 & 26.9 & 17.9 & 30.8 & 13.2 & 23.2 & 12.1 & 22.0 & 3.0 & 5.2 & 3.6 & 6.3 & 7.8 & 14.2 \\
\hline Ave. & 5.97 & 16.61 & 6.19 & 15.24 & 4.87 & 10.89 & 5.64 & 13.46 & 6.01 & 13.69 & 8.91 & 15.99 & 2.96 & 7.72 & 3.11 & 8.12 & 3.19 & 6.64 \\
\hline
\end{tabular}

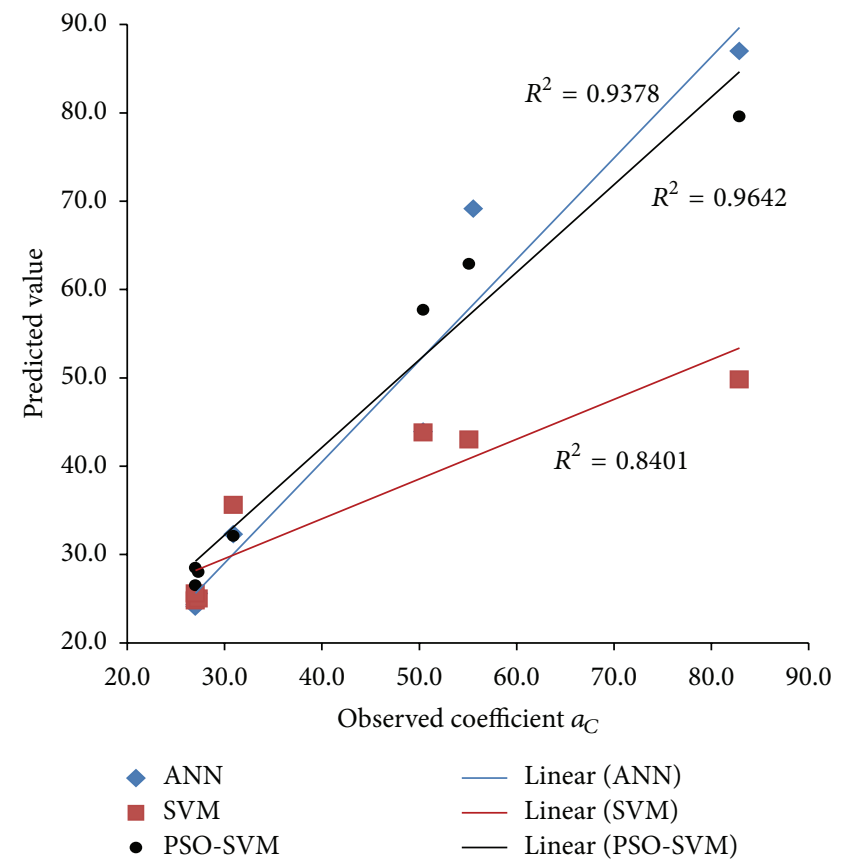

Figure 5: Predictive performance of $a_{C}$.

are optimized by PSO algorithm and then a very good predictive performance is obtained. Therefore, optimization of the parameters in SVM is critical to give a good predictive performance. And the PSO-SVM is proven to perform much better than the ANN and simple SVM in the estimation of ELC of sandstone.

As mention above, doing such fundamental experiments will cost too much and sometimes even quite difficult. The introduced methods for determining the elastic parameters of porous rock materials can in some sense be appropriate to obtain such parameter as the elastic compressibility values. If a certain number of samples have been done, then it is only needed to measure some related physical features and parameters that are easily tested. The elastic compressibility can be estimated with good accuracy using the PSO-SVM method. Therefore, this technique is feasible and could

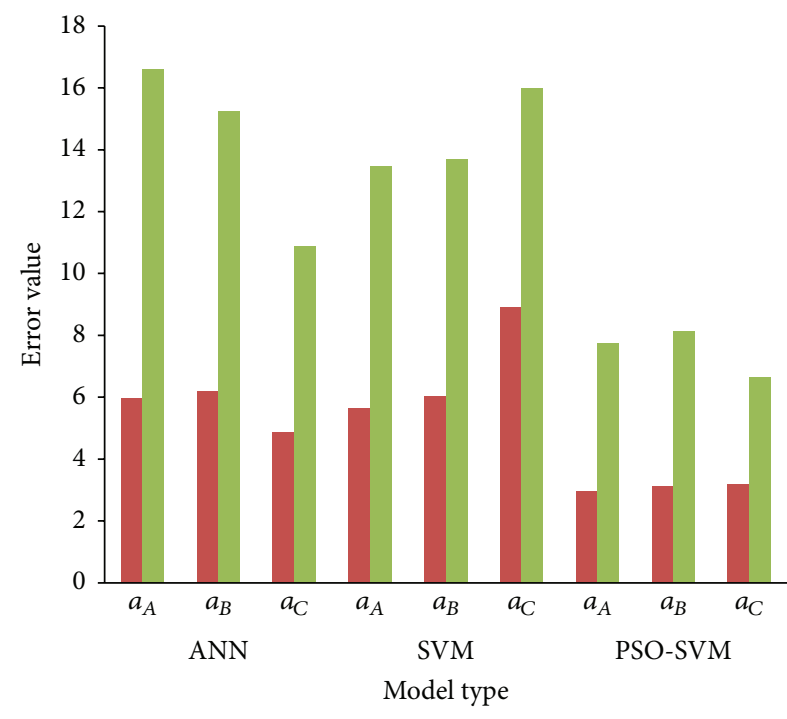

- Average absolute error

- Average relative error (\%)

FIGURE 6: Average predictive errors of the models.

be used as a potential tool for the estimation of elastic parameters of porous rock.

\section{Conclusions}

Based on the results obtained, conclusions can be made as follows.

(1) The elastic compressibility of sandstone is found to have linear relations with the rock minerals, applied pressure, and pores by a linear regression analysis. Other parameters are excluded by the stepwise regression and thus can be considered not vulnerable in the estimation.

(2) The predictive performances obtained by the ANN, SVM, and PSO-SVM prove that these techniques are feasible and appropriate for the estimation elastic compressibility of sandstone and can be applied to 
the estimation of other elastic parameters of porous rock material based on the mineral compositions and microstructural features.

(3) The PSO-SVM is found to have the best predictive performance among the applied models in the estimation. It can be used as an alternative potential tool for evaluation of many other parameters of rock materials.

Nevertheless, this approach is developed and implemented based on the collected data samples. The more the samples collected are the more accurate results this technique will produce. In future, this approach is to be validated by more data samples with variability features to show its generalization ability in the estimation of elastic parameters of porous rocks.

\section{Conflict of Interests}

The authors declare that there is no conflict of interests regarding the publication of this paper.

\section{Acknowledgments}

The present work was jointly supported by China Key Basic Research Program (973 Program, no. 2011CB013504) and China Natural Science Foundation (no. 11272114).

\section{References}

[1] R. Yoshinaka, M. Osada, H. Park, T. Sasaki, and K. Sasaki, "Practical determination of mechanical design parameters of intact rock considering scale effect," Engineering Geology, vol. 96, no. 3-4, pp. 173-186, 2008.

[2] W. Lin, Mechanical Properties of Mesaverde Sandstone and Shale at High Pressures, Lawrence livermore national laboratory, University of California, 1983.

[3] L. Caruso, G. Simmons, and R. Wilkens, "The physical properties of a set of sandstones-part I. The samples," International Journal of Rock Mechanics and Mining Science \& Geomechanics Abstracts, vol. 22, no. 6, pp. 381-392, 1985.

[4] A. Gens, A. Ledesma, and E. E. Alonso, "Estimation of parameters in geotechnical backanalysis-II. Application to a tunnel excavation problem," Computers and Geotechnics, vol. 18, no. 1, pp. 29-46, 1996.

[5] F. G. Bell, "The physical and mechanical properties of the fell sandstones, Northumberland, England," Engineering Geology, vol. 12, pp. 1-29, 1978.

[6] G. N. S. Koukis and S. Papanakli, "Laboratory testing properties of sandstones," in Proceedings of the 11th Internationa Congress of the Geological Society of Greece, pp. 1695-1699, Bulletin of the geological society of Greece, Athens, Ga, USA, 2007.

[7] M. E. Mostafa, F. Soliman, H. A. Ashry, and H. Helai, "Phyfical, electrical and mechanical properties of grantitic and limestone rocks with emphasis to their chemical compostions," Communications de la Faculté des Sciences de l'Université d'Ankara. Série C, vol. 12, pp. 1-10, 1994.

[8] P. Turgut, M. I. Yesilnacar, and H. Bulut, "Physico-thermal and mechanical properties of Sanliurfa limestone, Turkey," Bulletin of Engineering Geology and the Environment, vol. 67, no. 4, pp. 485-490, 2008.

[9] Y. Tan, D. Huang, and Z. Zhang, "Rock mechanical property influenced by inhomogeneity," Advances in Materials Science and Engineering, vol. 2012, Article ID 418729, 9 pages, 2012.

[10] K. Zorlu and K. E. Kasapoglu, "Determination of geomechanical properties and collapse potential of a caliche by in situ and laboratory tests," Environmental Geology, vol. 56, no. 7, pp. 1449$1459,2009$.

[11] Y. Zhang, J. He, Y. Wei, and D. Nie, "Prediction research of deformation modulus of weak rock zone under in-situ conditions," Journal of Mountain Science, vol. 8, no. 2, pp. 345353, 2011.

[12] R. A. Bearman, C. A. Briggs, and T. Kojovic, "The application of rock mechanics parameters to the prediction of comminution behaviour," Minerals Engineering, vol. 10, no. 3, pp. 255-264, 1997.

[13] S. Kahraman, "Evaluation of simple methods for assessing the uniaxial compressive strength of rock," International Journal of Rock Mechanics and Mining Sciences, vol. 38, no. 7, pp. 981-994, 2001.

[14] C. Gokceoglu, H. Sonmez, and A. Kayabasi, "Predicting the deformation moduli of rock masses," International Journal of Rock Mechanics and Mining Sciences, vol. 40, no. 5, pp. 701-710, 2003.

[15] A. Kayabasi, C. Gokceoglu, and M. Ercanoglu, "Estimating the deformation modulus of rock masses: a comparative study," International Journal of Rock Mechanics and Mining Sciences, vol. 40, no. 1, pp. 55-63, 2003.

[16] M. Calvello and R. J. Finno, "Selecting parameters to optimize in model calibration by inverse analysis," Computers and Geotechnics, vol. 31, no. 5, pp. 411-425, 2004.

[17] B.-S. Chun, W. R. Ryu, M. Sagong, and J.-N. Do, "Indirect estimation of the rock deformation modulus based on polynomial and multiple regression analyses of the RMR system," International Journal of Rock Mechanics and Mining Sciences, vol. 46, no. 3, pp. 649-658, 2009.

[18] N. Babanouri, H. Mansouri, S. K. Nasab, and M. Bahaadini, "A coupled method to study blast wave propagation in fractured rock masses and estimate unknown properties," Computers and Geotechnics, vol. 49, pp. 134-142, 2013.

[19] X. Feng, Introduction of Intelligent Rock Mechanics, Science Press, Beijing, China, 2000.

[20] Q. Zhang and J. Song, "Predicting mechanical behaviors of rock or rock engineering by neural network," Chinese Journal of Rock Mechanics and Engineering, vol. 11, no. 1, pp. 35-43, 1992.

[21] B.-R. Chen, X.-T. Feng, X.-L. Ding, and P. Xu, "Back analysis on rheological parameters based on pattern-genetic-neural network," Chinese Journal of Rock Mechanics and Engineering, vol. 24, no. 4, pp. 553-558, 2005.

[22] W.-Y. Xu, F. Xu, and D.-W. Liu, "Study and application of displacement time series forecast based on APSO-WLSSVM," Chinese Journal of Geotechnical Engineering, vol. 31, no. 3, pp. 313-318, 2009.

[23] Z.-B. Liu, W.-Y. Xu, F. Xu, and L.-W. Wang, "Mechanical parameter analysis of rock material via PLSR model," Key Engineering Materials, vol. 467-469, pp. 1826-1831, 2011.

[24] Z. Liu, W. Xu, and J. Shao, "Gaussian process based approach for application on landslide displacement analysis and prediction," Computer Modeling in Engineering \& Sciences, vol. 84, no. 2, pp. 99-122, 2012. 
[25] Z. Liu, J. Shao, W. Xu, and Y. Meng, "Prediction of rock burst classification using the technique of cloud models with attribution weight," Natural Hazards, vol. 68, no. 2, pp. 549-568, 2013.

[26] C. Cortes and V. Vapnik, "Support-vector networks," Machine Learning, vol. 20, no. 3, pp. 273-297, 1995.

[27] R. Gholami, A. R. Shahraki, and M. Jamali Paghaleh, "Prediction of hydrocarbon reservoirs permeability using support vector machine," Mathematical Problems in Engineering, vol. 2012, Article ID 670723, 18 pages, 2012.

[28] S. S. Keerthi, V. Sindhwani, and O. Chapelle, "An efficient method for gradient-based adaptation of hyperparameters in svm models," in Advances in Neural Information Processing Systems Cambridge, B. Schölkopf, J. Platt, and T. Hoffman, Eds., pp. 673-680, MIT Press, 2007.

[29] H. N. Hall, "Compressibility of reservoir rocks," Journal of Petroleum Technology, vol. 5, no. 1, pp. 17-19, 1953.

[30] G. H. Newman, "Pore-volume compressibility of consolidated, friable, and unconsolidated reservoir rocks under hydrostatic loading," Journal of Petroleum Technology, vol. 25, pp. 129-134, 1973.

[31] X. N. Xue and M. Li, "New calculating method of rock compressibility coeffcients petroleum geology and oilfield," Development in Daqing, vol. 30, no. 3, pp. 110-112, 2011.

[32] T. M. Wissler and G. Simmons, "The physical properties of a set of sandstones-Part II. Permanent and elastic strains during hydrostatic compression to $200 \mathrm{MPa}$," International Journal of Rock Mechanics and Mining Sciences and, vol. 22, no. 6, pp. 393406, 1985.

[33] R. W. Zimmerman, W. H. Somerton, and M. S. King, "Compressibility of porous rocks," Journal of Geophysical Research, vol. 91, no. 12, pp. 765-712, 1986.

[34] C. Li, X. Chen, and Z. Du, "A new relationship of rock compressibility with porosity," in Proceedings of the SPE Asia Pacific Oil and Gas Conference and Exhibition (APOGCE '04), pp. 163-167, October 2004.

[35] A. A. Jalalh, "Compressibility of porous rocks: part II. New relationships," Acta Geophysica, vol. 54, no. 4, pp. 399-412, 2006.

[36] A. Suman, Uncertainties in Rock Pore Compressibility and Sersmic History Matching, Stanford Stanford University, 2009.

[37] J. Kennedy and R. Eberhart, "Particle swarm optimization," in Proceedings of the IEEE International Conference on Neural Networks, pp. 1942-1948, December 1995.

[38] R. B. Darlington, Regression and Linear Models, Mcgraw-Hill College, New York, NY, USA, 1990.

[39] S. Awasthi, General Stepwise Regression (GSR), University of Texas at Arlington. 

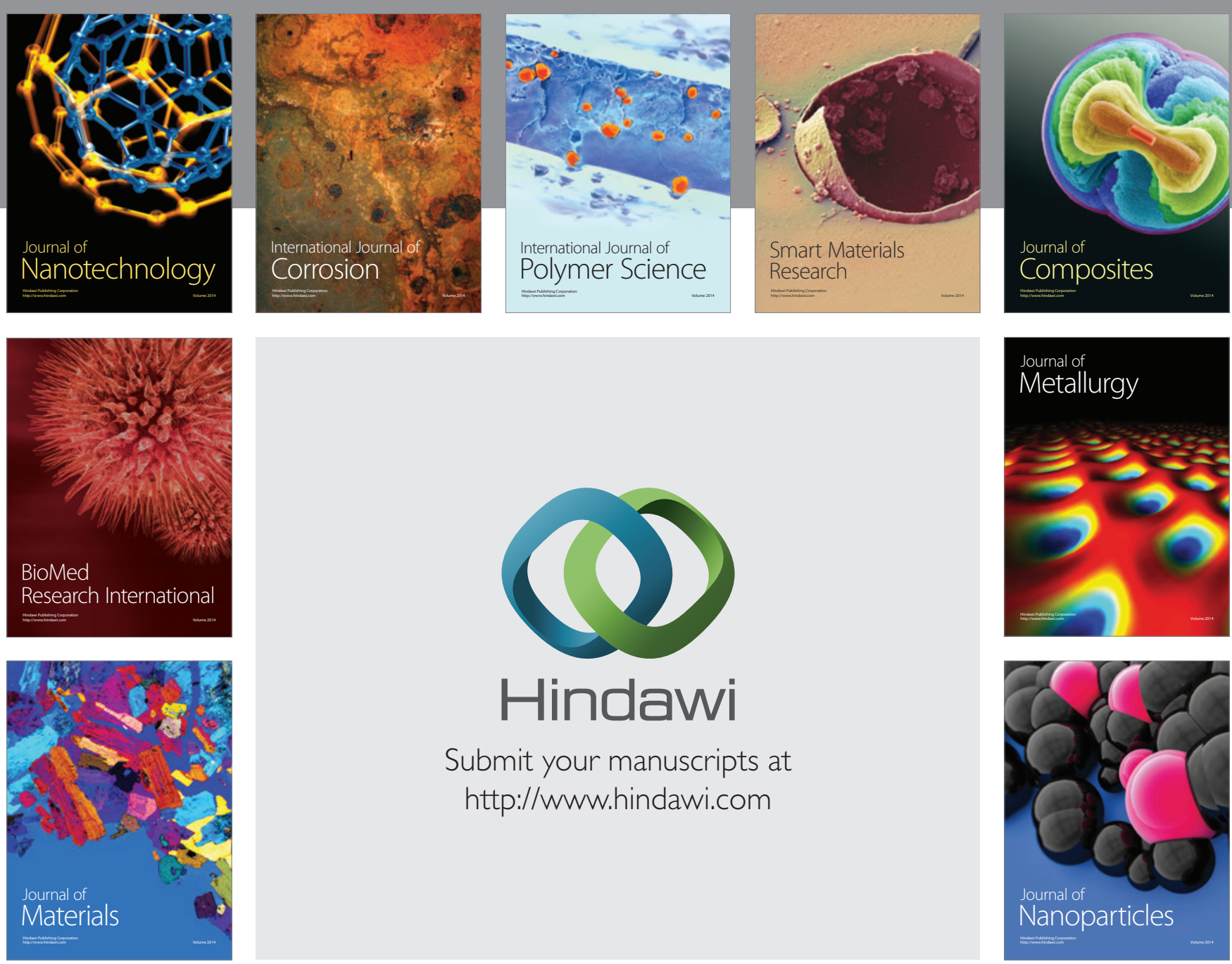

Submit your manuscripts at http://www.hindawi.com
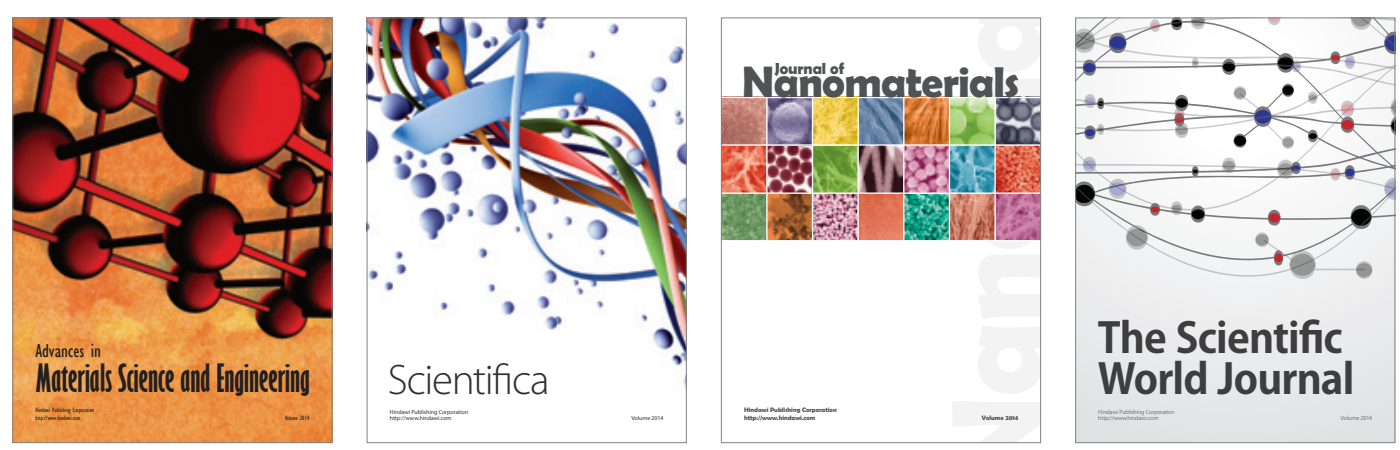

\section{The Scientific World Journal}
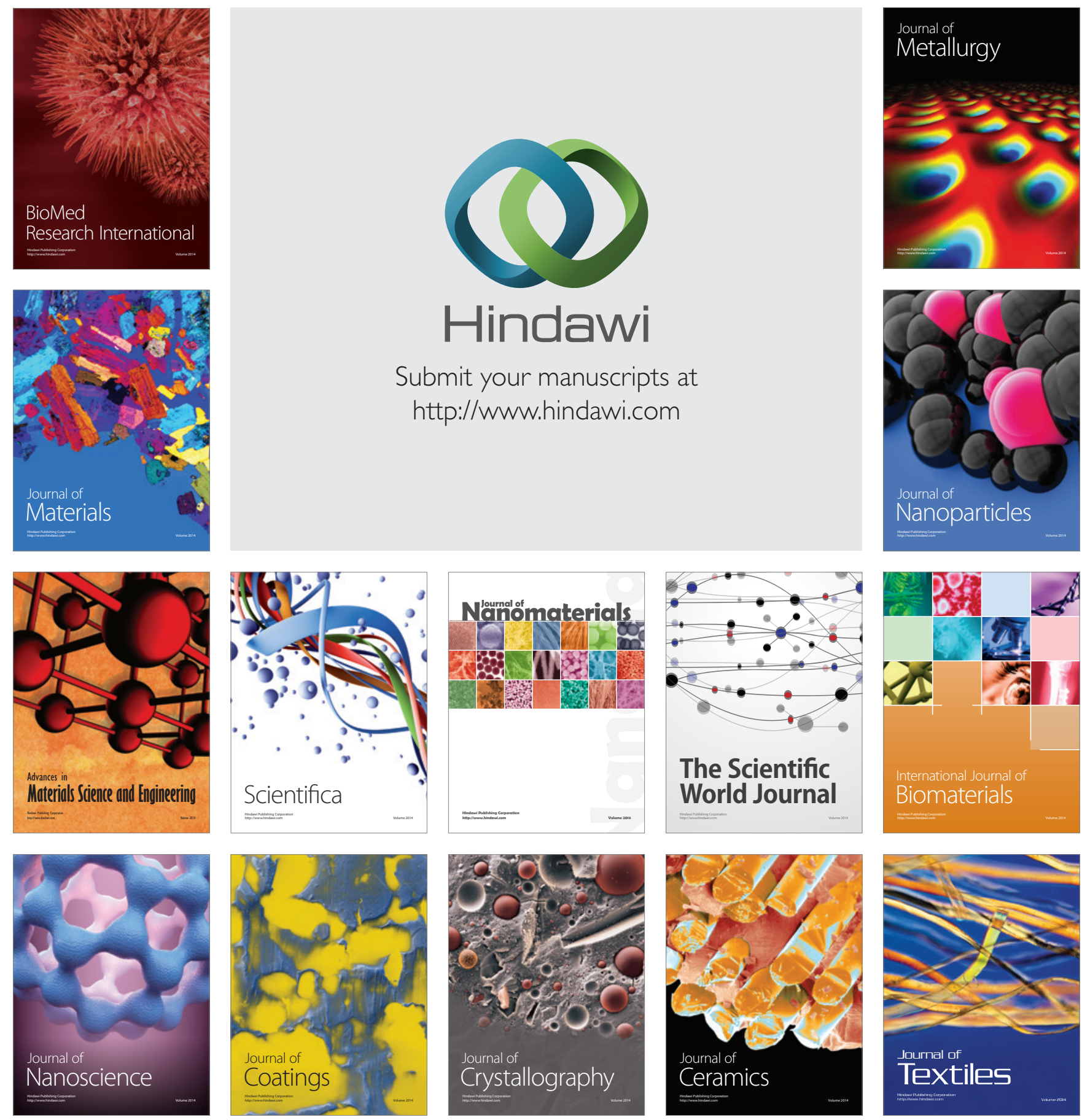